\title{
M-mode State Based Identification in Ultrasound Videos of the Atherosclerotic Carotid Plaque
}

\author{
C. P. Loizou, Member, IEEE, M. Pantziaris, and C. S. Pattichis, Senior Member, IEEE, E. Kyriakou, \\ Member, IEEE
}

\begin{abstract}
Monitoring the wall and plaque changes in the carotid artery (CA) can provide useful information for the assessment of the atherosclerotic disease. Using a motion mode (M-mode) image, detailed information may be obtained about wall and lumen dimensions, systolic and diastolic artery diameter and distension, wall and plaque motion and thickness, and also their corresponding states (timings). The wall thickness and the diameter of the $\mathrm{CA}$ change during the cardiac cycle are an indicator of regional contraction and therefore an indication of a disease. The objective of this work was to investigate how M-mode state based modeling of the $\mathrm{CA}$ can be derived from a B-mode ultrasound video. Briefly, 10 longitudinal CA ultrasound videos acquired from symptomatic subjects at risk of atherosclerosis were broken into frames and their M-mode images were generated. These were then despeckled and the atherosclerotic carotid plaque was segmented from each video, in order to extract the states of the video. By identifying the states of the $\mathrm{CA}$, we can distinguish between normal and abnormal plaque motion. It was shown in this work, that M-mode state based modeling derived from Bmode videos can be used successfully to derive the carotid states and assess the corresponding wall changes. However, further work in a larger number of videos is needed for validating the proposed method and to differentiate between normal and abnormal state based plaque motion analysis.
\end{abstract}

\section{INTRODUCTION}

$\mathrm{C}$ ARDIOVASCULAR disease (CVD) is the third leading cause of death and adult disability in the industrial world after heard attack and cancer. According to [1], 80 million American adults have one or more types of CVD of whom about half are estimated to be age 65 or older. The carotid artery (CA) function and its wall motion can be assessed in real time by ultrasound, when recording sequences of images. One approach for quantifying the wall changes would be to quantitatively assess wall motion in the images as they are acquired [2]. The objective of this work was to investigate how M-mode state based modeling of the CA can be derived from B-mode ultrasound video that will

Manuscript received January 15, 2010.

C.P. Loizou, is with the Department of Computer Science, School of Sciences, Intercollege, P.O.Box 51604, CY-3507, Limassol, Cyprus (phone: 357-25-381180; e-mail: loizou.c@ lim.intercollege.ac.cy; loizou.christos@ucy.ac.cy).

M. Pantziaris is with the Cyprus Institute of Neurology and Genetics, Nicosia, Cyprus; (e-mail: pantzari@cing.ac.cy).

C.S. Pattichis, is with the Department of Computer Science, University of Cyprus, Nicosia, Cyprus; (e-mail: pattichi@ucy.ac.cy).

E. Kyriakou, is with the Department of Computer Science, Frederick University, Limassol, Cyprus; (e-mail: e.kyriacou@frederick.ac.cy). subsequently facilitate the assessment of states and wall changes of the carotid. In this paper we focus on the states extraction.

M-mode images also allow for the analysis of regional wall motion dynamics, which can be used to diagnose myocardial ischemia [3]. Gradients between velocity values in different myocardial layers, computed using M-mode tissue Doppler images have also been shown to provide diagnostic information [4]. On 2D images, velocity profiles and their evolution along time can be obtained from any line of the heart selected by the user, a procedure usually known as anatomic M-mode [5]. In [6] M-mode images were generated from echocardiography images by manually selecting a line over the myocardium. In [7] the states of echocardiogram videos were modeled and extracted based on a hierarchical state based modeling by identifying the views, states and substates of objects. Finally, in [2] the diastolic and systolic diameters of the CA were estimated by using B-mode, M-mode and Hough transform and it was shown that those estimated by Hough transform are more accurate. The objective of our study was to generate an Mmode image from its respective CA B-mode video, extract its states and assess the wall changes.

In M-mode echocardiography images (or videos), high frequency modulation components are found in the vertical direction (changes in the images contrast), whereas frequency modulation in the horizontal direction is less significant. This observation was also utilized in [8], where an AM-FM demodulation was proposed, to track the wall boundaries of the heart. A filter was adaptively selected, which emphasizes the image's contrast change level at a given region of the image, to track the wall's boundaries.

We hypothesized that converting real time ultrasound video (see Fig. 1a) to an M-mode representation (see Fig. 1e), would allow (a) a simple continuous display of CA wall function, (b) simplify real time segmentation and automatic extraction of wall motion parameters such as the identification of the $\mathrm{CA}$ video states, and c) differentiate between normal and abnormal motion.

The paper is organized as follows. In section II materials and methods are given, in section III the results are presented, and sections IV and $\mathrm{V}$ give the discussion and the concluding remarks respectively. 
Step 1: Record and digitize the video of the CA.

Step 2: Load the initial B-mode video and despeckle all frames by applying the $l s m v$ despeckle filter [10].

Step 3: Segment the plaque and the opposite wall (every 5 frames) of the CA by using a snakes segmentation algorithm [9] (see Fig. 2b, c).

Step 4: Approximate the plaque by an ellipse and identify the minor and major axis. Define rays-lines that cross the major axis of the plaque, placed at the major axis quintiles $(20 \%, 40 \%, 60 \%$, and $80 \%)$ (see Fig. 2d).

Step 5: Read the same ray-line (one pixel wide) estimated in step 4 for each consecutive video frame Step 6: Generate the M-mode image by placing these lines (rays) in the Y-axis, and as X-axis each frame of the video (see Fig. 2e). Four M-mode images are thus generated, one for each ray of Fig. 1d).

Step 7: Manually locate the near and far walls on the M-mode image and binarise the image. Convert both areas to binary by image thresholding, in order to extract edges more easily. A threshold is calculated from the despeckled grayscale image according to [11] so that the intraclass variance of the thresholded black and white pixels is minimized.

Step 8: Dilate the binary image (of step 7) by an $3 \times 3$ pixel-structuring element consisting of ones. This morphological operation is performed to close small gaps and form a continuous boundary.

Step 9: Remove erroneous small edges that might trap the snake. This is carried out by labeling connecting components in the image where the number of connecting components in a pixel neighborhood was chosen to be eight. Small segments that are smaller than 20 pixels, and do not belong in the boundary are therefore removed.

Step 10: Extract both the near and far wall boundaries and construct the corresponding interpolating Bspline. Sample the interpolating B-splines, in equal distance points, in order to define a number of snake elements on the contour. The number of snake points was variable and was determined according to the area chosen by the user.

Step 11: Map the detected contour points from step 10, on the M-mode image of Fig. 2e, to form the initial snake contours for the near and far walls (see Fig. 2f).

Step 12: Apply the snakes segmentation algorithm [9] to extract and refine the final snake contours for the near and far walls for the 4 rays of Fig. 1d) (see Fig. 2g).

Step 13: Draw the lumen rate of change (see Fig. 2h), i.e. difference between the near and far wall boundaries (see Fig. 2g).

Step 14: Compute the average lumen rate of change for the four rays estimated in step 13 of Fig. $2 \mathrm{~h}$, see Fig. 2i. Estimate minima and maxima on the averaged lumen rate of change curve for each cardiac cycle. Mark each minimum with a cross (+) that indicates minimum carotid diameter during contraction, and each maximum with an asterisk $\left(^{*}\right)$ that indicates maximum carotid diameter during distension. These markings describe the M-mode states during a cardiac cycle.

Fig. 1. Steps followed in the analysis of ultrasound videos for generating the M-mode state based identification.

\section{MATERIALS AND METHODS}

Figure 1 summarizes all the steps followed in the analysis of ultrasound videos for generating the M-mode state based identification.

\section{A. Recording of CA Videos (Fig. 1, step 1)}

A total of $10 \mathrm{CA}$ digitized videos from B-mode longitudinal ultrasound segments were recorded by the ATL HDI-5000 ultrasound scanner (Advanced Technology Laboratories, Seattle, USA) at a frame size of $576 \times 768$ pixels with 256 gray levels, a spatial resolution of 16.6 pixels per $\mathrm{mm}$ (i.e. the resolution is $60 \mu \mathrm{m}$ ), and having a frame-rate of 20 frames per second. For detailed technical characteristics of the ultrasound scanner (multi element ultrasound scan head, operating frequency, acoustic aperture, spatial pulse length, axial system resolution, and transmission focal range) refer to [9].

The videos were recorded at the Cyprus Institute of Neurology and Genetics, in Nicosia, Cyprus, from 10 (female/male) symptomatic patients aged between 26 and 85 years old, with a mean age of 54 years. These subjects were at risk of atherosclerosis and they have already developed clinical symptoms.

\section{B. Speckle Reduction Filtering (see Fig. 1, step 2)}

Due to the presence of speckle noise in ultrasound video, it is difficult to effectively apply different image processing and analysis algorithms. Speckle is a multiplicative locally correlated noise, which limits the contrast resolution in 
ultrasound imaging affecting the detectability of small, low contrast lesions. It was therefore recommended in [9], [10] to remove speckle noise from the B-mode image prior to further analysis. In this study, the linear scaling filter (linear scaling mean variance-lsmv) [10] utilizing the mean and the variance of a pixel neighborhood was used. The filter may be described by a weighted average calculation using sub region statistics to estimate statistical measurements over $5 \times 5$ pixel windows applied for three iterations [10]. The filter was applied in each consecutive video frame.

\section{Snakes Segmentation (See Fig. 1, step 3)}

Segmentation of the CA in the upper and lower walls (Fig. 2b) in the first frame of the CA video was carried out after despeckle filtering [10] every 5 frames, using the semi automated snakes segmentation system proposed and evaluated on ultrasound images of the CA in [9], which is based on the Williams \& Shah [12] snake. The plaque (either in the upper or lower wall) was then approximated by an ellipse and the minor and major axis were identified (see Fig. 2c).

\section{M-mode Image Generation (See Fig. 1, step 6)}

The M-mode image could be generated in such a manner that it crosses the whole plaque borders having maximum motion in opposite directions [7]. Perpendicular lines that cross the major axis of the plaque were placed automatically at the major axis quintiles $(20 \%, 40,60 \%$, and $80 \%$ ) (see Fig. 2d). By scanning the intensity values along the straight perpendicular line selected by the user, the M-mode image is generated, by positioning this line in parallel with the $\mathrm{Y}$-axis for each consecutive frame of the video that appear in the Xaxis. Four M-mode images were generated for each of the corresponding four perpendicular lines. The manual delineations as well as all other measurements were performed using a system implemented in MATLAB ${ }^{\circledR}$ from our group.

\section{E. Near and far wall boundary extraction and rate of} change (see Fig. 1, steps 7-12)

The M-mode image, (see Fig. 1, step 6) were converted to binary, and morphological operators were applied to smooth the edges (see Fig. 1, steps 7-10). Then, edge detection was applied on each M-mode image in order to derive the initial near and far wall boundaries (see Fig. 1 step 11, as illustrated in Fig. 2f). The snakes segmentation system [9] was also used to refine the derived snakes contours (see Fig. 1 step 12, as illustrated in Fig. 2g) for the near and far walls of the CA found on the M-mode image.

\section{F. State identification (see Fig. 1, steps 13-14)}

Compute the lumen rate of change (see Fig. $2 \mathrm{~h}$ ), i.e. the difference between the near and far wall boundaries (of Fig. 2g) (see Sig. 1 step 13). The above procedure is applied for the 4 rays. Compute the average lumen rate of change for the four rays estimated in step 13 of Fig. $2 \mathrm{~h}$, see Fig. $2 \mathrm{i}$ (see

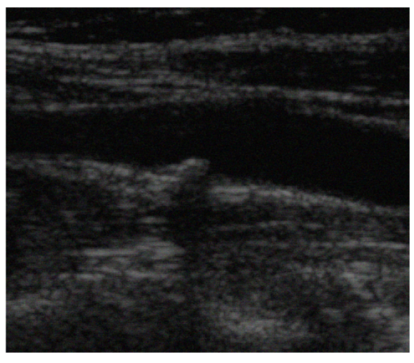

(a)

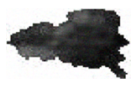

(c)

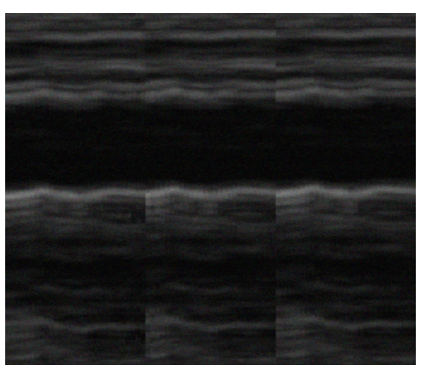

(e)

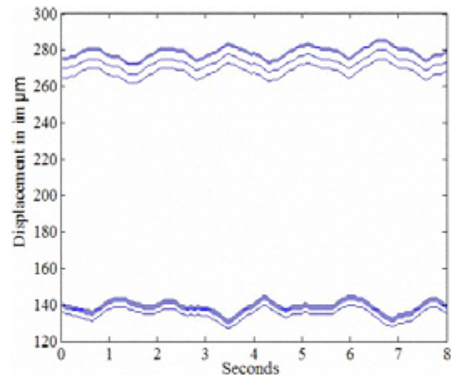

(g)

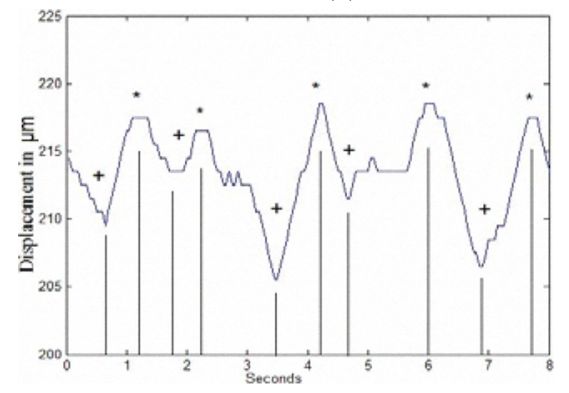

(i)

Fig. 2. Illustration of the M-mode state based identification. (a) Ultrasound video of the CA (first frame of video). (b) Segmentation of the plaque and the near wall of the CA by snakes. (c) Extracted plaque. (d) Quintile ray definition on the segmented plaque (4 rays). (e) Despeckled M-mode image generated form the CA video for ray 2. (f) Segmented near and far wall boundaries for ray 2. (g) Near and far wall boundary movements for the four rays of Fig. $1 \mathrm{~d}$. The $\mathrm{x}$-axis shows the time in seconds and the $y$-axis shows the displacement in micrometers. (h) Lumen rate of change for the 4 rays (difference between near and far wall boundaries of Fig. 1 g. (i) Average rate of change for the 4 rays of Fig. $1 \mathrm{~h}$. Cross $(+)$ indicates minimum carotid diameter during contraction, and asterisk $(*)$ indicates maximum carotid diameter during distension. 
Fig.1 step 14). Estimate minima and maxima on the averaged lumen rate of change curve for each cardiac cycle. Mark each minimum with a cross $(+)$ that indicates minimum carotid diameter during contraction, and each maximum with an asterisk (*) that indicates maximum carotid diameter during distension. These markings describe the M-mode states during a cardiac cycle.

\section{G. Evaluation metrics}

In order to evaluate our algorithm, the following metrics between the automated and manual state diagram timings were computed:

1) The randomized mean square error (RMSE):

$$
R M S E={\sqrt{\sum_{i}\left|A_{i}-M_{i}\right|}}^{2} /(2 N)
$$

where $\mathrm{A}$ and $\mathrm{M}$ represent the automated and manual state diagram timings, and $\mathrm{N}$ is the number of states $(2 \mathrm{x}$ the heart rhythm).

2) The normalized mean square error (NMSE):

$$
N M S E=100 * R M S E / s^{*} d_{N}
$$

with $\operatorname{std}_{\mathrm{N}}$ is the standard deviation over all timings.

3) The mean average error (MAE):

$$
M A E=\sum_{i}\left|A_{i}-M_{i}\right| / N
$$

4) The mean average randomized error:

$$
M A R E=\sum_{i}\left|\frac{A_{i}-M_{i}}{M_{i}}\right| / N .
$$

\section{RESULTS}

Figure 2 illustrates the M-mode and the states generation from a CA video. Table I presents the results of the evaluation metrics between the manual and the automated state timings (average \pm std) in microseconds, for all 10 videos of the CA. Table II presents the results of the carotid diameter during contraction (CDC) and the carotid diameter during distension (CDD) ( $\mathrm{CDC}=5.26 \pm 0.52 \mathrm{~mm}$, $\mathrm{CDD}=5.81 \pm 0.59 \mathrm{~mm}$ ), and the percentage of the carotid wall distension $\quad\left(\% \mathrm{CWD}=((\mathrm{CDD}-\mathrm{CDC}) / \mathrm{CDC})^{*} 100 \%\right)$, which was $\% \mathrm{CWD}=9.21 \pm 3.88 \%$.

\section{DISCUSSION}

The objective of this work was to investigate how Mmode state based modeling of the CA can be derived from a B-mode ultrasound video. Briefly, 10 longitudinal CA ultrasound videos acquired from symptomatic subjects at risk of atherosclerosis were broken into frames and their Mmode images were generated. These were then despeckled and the atherosclerotic carotid plaque was segmented from each video, in order to extract the states of the video. By identifying the states of the $\mathrm{CA}$, we can distinguish between normal and abnormal plaque motion.

We have shown in this paper that state based video modeling can be used to identify video segments dynamic behavior in ultrasound videos of the CA.
TABLE I

EVALUATION METRICS BETWEEN THE MANUAL AND THE AUTOMATIC STATES IN $\mu$ secs.

\begin{tabular}{lcccc}
\hline \hline Video & RMSE & NRMSE & MAE & MARE \\
\hline 1 & 200 & 361 & 249 & 5 \\
2 & 309 & 408 & 349 & 1 \\
3 & 221 & 249 & 208 & 10 \\
4 & 184 & 211 & 169 & 6.5 \\
5 & 212 & 305 & 184 & 6.5 \\
6 & 279 & 386 & 142 & 6.5 \\
7 & 190 & 445 & 219 & 8.5 \\
8 & 391 & 431 & 433 & 11.5 \\
9 & 159 & 231 & 167 & 13.5 \\
10 & 143 & 195.50 & 111 & 9.50 \\
\hline Average & $228 \pm 76$ & $322 \pm 96$ & $222 \pm 99$ & $7.9 \pm 3.5$ \\
\hline \hline
\end{tabular}

RMSE: Relative mean square error, NRMSE: Normalized mean square error, MAE: Mean Absolute error, MARE: Mean absolute relative error.

TABLE II

CAROTID DiAMETER DURING CONTRACTION (CDC), CAROTID DIAMETER DURING DISTENSION (CDD) AND \% OF CAROTID WALL DISTENSION (\%CWD)

\begin{tabular}{llcc}
\hline \hline Video & CDC & CDD & \%CWD \\
\hline 1 & 4.59 & 4.92 & 7.19 \\
2 & 6.20 & 6.53 & 5.32 \\
3 & 6.10 & 6.65 & 9.02 \\
4 & 4.78 & 5.12 & 7.11 \\
5 & 4.96 & 5.12 & 3.23 \\
6 & 5.25 & 6.10 & 16.19 \\
7 & 5.23 & 5.84 & 11.66 \\
8 & 5.10 & 5.88 & 15.29 \\
9 & 5.28 & 5.89 & 11.55 \\
10 & 5.10 & 5.95 & 16.67 \\
\hline Average & $5.26 \pm 0.52 \mathrm{~mm}$ & $5.81 \pm 0.59 \mathrm{~mm}$ & $10.32 \pm 4.71 \%$ \\
\hline \hline
\end{tabular}

Golemati et al. [13], used block matching-based techniques to estimate arterial motion from B-mode CA ultrasound images. They estimated motion at the wall-lumen interface and within the tissue using block sizes of $3.2 \times 2.5$ $\mathrm{mm}$ and $6.3 \times 2.5 \mathrm{~mm}$, respectively, and the normalized correlation coefficient as the matching criterion. Measurements in 9 normal (nonatherosclerotic) adults showed that arterial wall distensibility in the radial direction was significantly higher than distensibility in the longitudinal direction $(10.2 \pm 4.5 \%$ vs. $2.5 \pm 0.89 \%)$. Results in [13] were very similar with our results, where we found in this study a distensibility of $9.21 \pm 3.88 \%$ in the radial direction.

In [14], a review was attempted regarding the analysis and quantification of motion of the CA wall from sequences of B-mode ultrasound images. Motion of the carotid artery wall and plaque from sequences of ultrasound images has been estimated using block matching and optical flow techniques. The main sources of arterial wall motion were also described and the most common techniques for CA wall motion were discussed.

In another study [15] it was shown that M-mode ultrasound analysis of circumferential inter-adventitial strain 
is a simple and safe non invasive tool for bed-side determination of CA mechanics. It proved to be a sensitive tool for measurements of strain decrease with increasing age and male sex and strain increase with smoking and obesity when evaluated on the right $\mathrm{CA}$. Therefore, it might be used for screening of "unsuccessful vascular aging" and still potentially reversible subclinical carotid atherosclerosis thereby lowering the cut-off value of vascular damage towards the values of unaffected population. It provides opportunities for introduction of different therapeutic or lifestyle change strategies for reduction or retardation of overt clinical manifestation of cerebrovascular disease.

In a recent study [7], the states of echocardiogram videos were identified based on a state detector, which first detects the view boundaries using histogram based comparison and edge change ratio. Next, a state classifier recognizes each view type by considering signal properties of different views. Then, state information is extracted from each view segment using a new type of M-mode generation method named as sweep M-mode. Finally, sub-states are extracted from colour flow Doppler images using Radial M-mode. The accuracy of the classifier was $97.19 \%$ which was higher than two existing approaches [16], [17]. Misclassification error of state detection was less than $13 \%$ which is reasonably low.

In [4] gradients between velocity values in different myocardial layers were computed using M-mode tissue Doppler images. It was shown that there is a non-uniform distribution of velocities in the different myocardial layers under normal conditions. This distribution of velocities undergoes a significant change in patients with ischemic myocardial damage. Intramyocardial wall motion analysis could have therefore, clinical applications in both the early detection of ischemia and myocardial viability.

In [6] M-mode from echocardiogram videos were generated, where the user manually selects a line in the first frame of the video sequence, where the trace was moved either manually or automatically, by maintaining it in the middle of the cardiac cavity along the sequence. In [3] a methodology and a software package developed to quantify M-mode tissue Doppler imaging, defining a number of quantitative parameters drawn from velocity and gradient curves obtained after segmenting the myocardial wall into anatomical layers was presented. These parameters were used to provide predictive clinical value to detect motion abnormalities in the presence of ischemia where 17 healthy and 18 ischemic patients were compared. It was shown that any single parameter related to the gradient intensity, particularly the maximum gradient at the moment of the ewave, provides meaningful clinical information, achieving a rate of correct classification of $79.1 \%$ on the same data set used for the analysis.

Normal and abnormal motion was also investigated in [5], where it was shown that measurement of systolic thickening using anatomical M-mode echocardiography offers an objective method to quantify systolic thickening at dobutamine echocardiography but has limited clinical feasibility.

Motion of the CA wall and plaque, which contributes to plaque rupture and occurrence of vascular symptoms, can be efficiently estimated from B-mode ultrasound image sequences using block matching or optical flow. The existing literature on the analysis of motion of the CA wall from video ultrasound is relatively limited probably due to the difficulties inherent in reliable acquisition of image data. Possible research perspectives in this field may be orientated towards to the development of new m-mode identification algorithms, where comparisons of the performance and results of different algorithms are crucial for our understanding of the motion field of the arterial wall. Furthermore, from a physiological viewpoint, the motion field of the healthy and diseased arterial wall remains to be studied in detail using one or more appropriate algorithms.

\section{CONCLUDING REMARKS}

We have shown in this paper that the proposed statebased video data model can be used efficiently to retrieve video segments depicting specific dynamic behaviour of the CA. Further work in more videos is needed to differentiate between normal and abnormal state based plaque motion analysis.

\section{REFERENCES}

[1] American Heart Association, Heart disease and stroke statistics-2009, update. Dallas, Texas, 2009.

[2] S. Golemati, J. Stoisis, T. Balkizas, K.S. Nikita "Comparison of Bmode, M-mode and Hough transform methods for measuring of arterial diastolic and systolic diameters," $27^{\text {th }}$ Ann. Conf. Proc. IEEE Eng. $M B E C$, pp. 1758-1761, 2005.

[3] M. Desco, M.J. Ledesma-Carbayo, E. Perez, A. Santos, J.C. Antoranz, N. Malpica, M.A. Garcia-Fernandez, "Assessment of normal and ischemic myocardium by quantitative m-mode tissue Doppler imaging", Ultras. In Med. Biol., vol. 18, pp. 561-569, 2002.

[4] P. Marcos-Alberca, M.A, Garcia-Fernandez, M.J. Ledesma, N. Malpica, A. Santos, M. Moreno, J. Bermelo, J.C. Antoranz, M. Desco, "Intramyocardial analysis of regional systolic and diastolic function in ischemic heart disease with Doppler tissue imaging; role of the different myocardial layers," J. Am. Soc. Echocardiogr., vol. 15, pp. 99-108, 2002.

[5] D. Mele, I. Pedini, P. Alboni, R.A. Levine, "Anatomic m-mode: a new technique for quantitative assessment of left ventricular size and function," Am. J. of Cardiol., vol. 81, pp. 82G-85G, 1998.

[6] G.R. Sutherland, T. Kukulski, J.U. Voight, J. D'Hooge, "Tissue Doppler echocardiography: Future developments," Echogardiogr., vol. 16, pp. 509-520, 1999.

[7] A. Roy, S. Sural, J. Mukherjee, A.K. Majunmdar, "State based modelling and object extraction from echocardiogram video," IEEE Trans. Inform. Techn. In Biomed., no.3, vol. 12, pp. 366-376, 2008.

[8] P. Rodriquez, M.S. Pattichis, M.B. Goens, "M-mode echogrardiography image and video segmentation based on AM-FM demodulation techniques," Proc. $25^{\text {th }}$ Ann. Int. Conf. IEEE EMBS, pp. 1176-1179, 2003.

[9] C.P. Loizou, C.S. Pattichis, M. Pantziaris, A. Nicolaides, "An integrated system for the segmentation of atherosclerotic carotid plaque," IEEE Trans. on Inform. Techn. in Biomedicine," vol. 11, no. 5, pp. 661-667, Nov. 2007.

[10] C. P. Loizou, C. S. Pattichis, C. I. Christodoulou, R. S. H. Istepanian, M. Pantziaris, and A. Nicoliades, "Despeckle filtering in ultrasound imaging of the carotid artery," IEEE Trans. Ultras. Ferr. Freq. Cont., vol. 52, no. 2, pp. 1653-1669, 2005. 
[11] N. Otsu, "A Threshold Selection Method from Gray-Level Histograms", IEEE T. Syst. Man. Cy., no. 9, vol. 1, pp. 62-66, 1979.

[12] D.J. Williams, M. Shah, "A fast algorithm for active contour and curvature estimation", GVCIP: Image Underst., vol. 55, no. 1, pp. 426, 1992.

[13] S. Golemati, A .Sassano, M.J. Lever, A.A. Bharath, S. Dhanjil, A.N. Nicolaides, "Motion analysis of carotid atherosclerotic plaque from Bmode ultrasound," Ultras. Med. Biol., vol. pp. 29: 387-399, 2003.

[14] S. Golemati, J. Stoitsis, K. S. Nikita, "Motion analysis of the carotid artery wall and plaque using B-mode ultrasound", Vasc. Diseas. Prevent., vol. 4, no. 4, pp. 296-302, 2007.

[15] M.-J. Jurasic, A. Lovrencic-Huzjan, M.R. Bedekovic, V. Demarin, "How to monitor vascular aging with an ultrasound," Journal of the Neurological Sciences, vol. 257, pp. 139-142, 2007.

[16] S. Ebadollahi, S. F. Chang, and H. Wu, "Automatic view recognition in echocardiogram videos using parts-based representation." Proc. IEEE Comp. Soci. Conf. on Comp. Vision and Pattern Recog., vol. 2, pp. 2-9, June 2004.

[17] S. Kevin, J. H. Park, B. Georgescu, C. Simopoulos, J. Otsuki, and D. Comaniciu, "Image-based multiclass boosting and echocardiographic view classification."Proc. IEEE Comp. Soci. Conf. on Comp. Vision and Pattern Recog., vol. 2, pp. 1559-1565, June 2006. 